\title{
Estudio de la señal de salida y diseño de la red de polarización de tubos fotomultiplicadores
}

\author{
L. Horacio Arnaldi \\ Laboratorio Detección de Partículas y Radiación \\ Centro Atómico Bariloche \\ S. C. de Bariloche, RN, Argentina \\ arnaldi@cab.cnea.gov.ar \\ Recibido: 07/05/19; Aceptado: 28/09/19
}

Study of the output signal and design of the polarization network of photomultiplier tubes

\begin{abstract}
Resumen-An analysis of the factors involved in the signal formation of the photomultiplier tubes is performed. These devices are extremely sensitive light detectors, widely used in photon counting applications. The study and design of the polarization network of photomultiplier tubes is discussed, laying the groundwork for performance testing. Through a detailed analysis of the operation of the photomultiplier tubes and their polarization electronics, it is expected that the user can separate the response of the polarization circuit from the information carried by the output pulses.
\end{abstract}

Keywords: photomultiplier tube; polarization network; photon counting.

Resumen- Se realiza un análisis de los factores intervinientes en la formación de la señal de los tubos fotomultiplicadores. Estos dispositivos son detectores de luz extremadamente sensibles, muy utilizados en aplicaciones de conteo de fotones. Se aborda el estudio y diseño de la red de polarización de los tubos fotomultiplicadores, sentando las bases para pruebas de desempeño. A través del análisis detallado del funcionamiento de los tubos fotomultiplicadores y su electrónica de polarización, se espera que el usuario pueda separar la respuesta del circuito de polarización de la información que llevan los pulsos de salida.

Palabras clave: tubo fotomultiplicador; red de polarización; conteo de fotones.

\section{INTRODUCCIÓN}

La aplicación del conteo de fotones en la detección de radiación y la espectroscopia sería imposible sin la existencia de dispositivos para convertir la salida de luz extremadamente débil de un pulso de centelleo en una señal eléctrica correspondiente. El tubo fotomultiplicador (o PMT, por photomultiplier tube) realiza esta tarea de manera remarcable, convirtiendo las señales de luz, que típicamente consisten en no más que una centena de fotones, en un pulso de corriente utilizable [1].

Los tubos fotomultiplicadores son detectores de luz de alta ganancia y bajo ruido. Se los puede utilizar desde el UV cercano hasta la región de rayos $\mathrm{X}$, y también como detectores de partículas. Un PMT está formado por un tubo de vidrio cerrado, cuya forma y tamaño varía según el modelo. En uno de los extremos está recubierto por dentro por una lámina delgada, denominada fotocátodo. Los fotones que golpean el fotocátodo expulsan electrones debido al efecto fotoeléctrico. Los fotoelectrones emitidos desde el fotocátodo hacia el interior del tubo son acelerados por un campo eléctrico hasta que alcanzan el primer dínodo y producen la emisión de electrones secundarios. Estos electrones secundarios luego inciden en el siguiente dínodo para producir emisiones adicionales de electrones secundarios. Este proceso se repite a través de las sucesivas etapas de dínodos (en cascada), cada uno de los cuales proporciona una ganancia de electrones de aproximadamente 4 a 5 . De esta forma se consiguen altas ganancias (del orden de $10^{6}$ a $10^{7}$ ). Por lo tanto una muy pequeña corriente fotoeléctrica del fotocátodo se puede observar como una gran corriente de salida en el ánodo del PMT. En la Figura 1 se puede ver un ejemplo de la disposición de las diferentes partes que componen un PMT y su red de polarización.

Desde las primeras aplicaciones de conteo de fotones, en la década de 1950, ha habido un rápido aumento en el uso de los PMT como detectores sensibles en diferentes disciplinas. Las técnicas de luminiscencia se utilizan ampliamente en las pruebas clínicas médicas [2] y de detección de drogas y en la inspección de la industria alimentaria para detectar antibióticos, insecticidas y bacterias [3], [4]. La dispersión láser se utiliza en la espectroscopia Raman para el análisis molecular y en el dimensionamiento de partículas submicrométricas [5], [6]. Un ejemplo de uso en el estudio de la física de partículas se puede encontrar en [7], donde la luz Cherenkov emitida por las partículas (principalmente fotones, electrones y muones) en el agua es detectada por tres tubos fotomultiplicadores colocados en la parte superior de cada tanque. También es muy común encontrar detectores basados en materiales centelladores y PMTs trabajando en conjunto. Los contadores de centelleo se utilizan para medir la radiación en una variedad de aplicaciones que incluyen monitoreo personal y ambiental de contaminación radiactiva, imágenes médicas, ensayos radiométricos, seguridad nuclear y seguridad de plantas nucleares.

A pesar de todos los usos enumerados, sin embargo, existe poca o muy dispersa información sobre cómo polarizar correctamente los PMTs y, dependiendo de la aplicación, sobre el tratamiento eléctrico que debe darse a la señal de salida. Este artículo aborda el estudio de la red de polarización de tubos fotomultiplicadores y cuáles son las características relevantes a tener en cuenta cuando se diseña para un uso específico. Se realiza un estudio detallado para una aplicación de conteo de fotones, en el cual el PMT debe 
trabajar con un centellador. Este es uno de los usos más demandantes, ya que el PMT debe responder a pulsos ultrarápidos $(\sim \mathrm{ns}) \mathrm{y}$, en general, poco espaciados en el tiempo uno del otro.

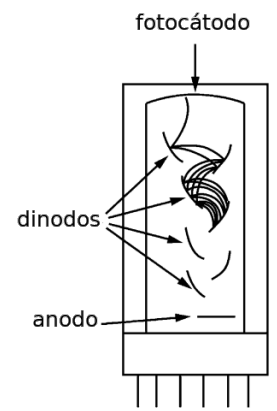

(a)

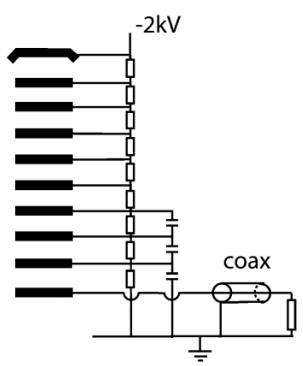

(b)
Figura 1. (a) Representación esquemática de un tubo fotomultiplicador y su geometría de electrodos. (b) Divisor de voltaje para proveer el potencial adecuado al cátodo y a los dínodos.

\section{CONSIDERACIONES GENERALES}

La polarización de un PMT puede realizarse usando una fuente de tensión independiente para cada etapa, pero resulta más conveniente usar una red divisora de tensión consistente en una serie de resistores entre tierra y un potencial elevado. La corriente que fluye por esta red divisora establece una serie de potenciales que son aplicados a los dínodos y a los elementos de enfoque del fotomultiplicador. Estos potenciales crean los campos electrostáticos necesarios para enfocar los electrones sobre el primer dínodo y para acelerar la cascada de electrones entre los sucesivos dínodos, proveyendo así una amplificación de corriente. Una red divisora ideal es aquella que mantiene los potenciales de los dínodos constantes independientemente de la corriente de salida. En la práctica resulta dificultoso satisfacer esas necesidades.

Las redes de polarización de PMTs que están diseñadas para aplicaciones de uso general no suelen ser apropiadas para el conteo de fotones. Este tipo de redes no permiten altas tasas de conteo, con frecuencia causan problemas de doble conteo y entregan características de plateau ${ }^{1}$ pobres. Si la aplicación requiere buena resolución de tiempo y buen desempeño para el conteo de fotones se hace necesario recurrir a un diseño más cuidado de la red de polarización, a menudo en configuración cónica ("tapered") y con blindaje magnético correcto.

Uno de los datos que suelen informar los fabricantes es la eficiencia cuántica del PMT, $\eta(\lambda)$, a una determinada longitud de onda. La eficiencia cuántica es un parámetro importante en el conteo de fotones; indica la probabilidad de emisión de fotoelectrones cuando un solo fotón golpea el fotocátodo, es función de la longitud de onda $\lambda$ del fotón incidente y normalmente se expresa como un porcentaje. Consideraciones prácticas importantes tales como la resolución y la relación señal a ruido $(\mathrm{S} / \mathrm{N})$ están

\footnotetext{
${ }^{1}$ Juntas, las características de ganancia del PMT y el umbral de discriminación elegido definen una meseta (plateau) de conteo dentro de la cual la tasa de conteo debido a un flujo de radiación constante varía muy poco en función de la alta tensión aplicada al tubo. Tal meseta se describe generalmente en términos de su pendiente (en porcentaje de cambio de tasa de conteo por voltios) y su excursión en voltios.
}

relacionados a la eficiencia cuántica. $\eta(\lambda)$ aparece como un factor multiplicativo en la ecuación que describe la tensión de salida del PMT. A modo de ejemplo, la Figura 2 muestra las características de sensibilidad cuántica como función de la longitud de onda de los fotones incidentes para el PMT modelo R1463, de Hamamatsu [8].

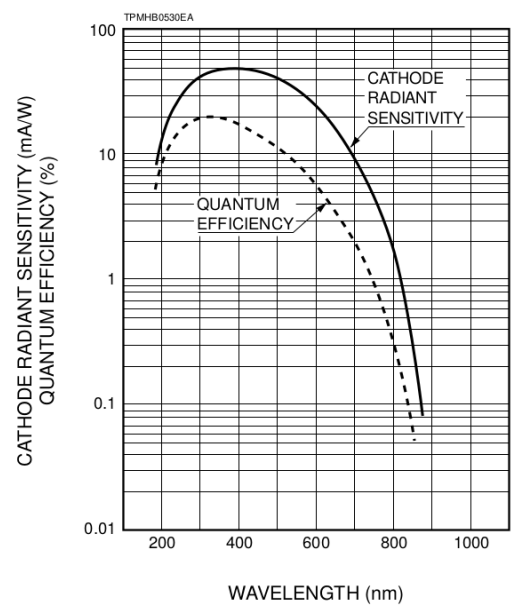

Figura 2. Características de sensibilidad cuántica para el tubo fotomultiplicador modelo R1463 de Hamamatsu [8].

\section{II-A. Ganancia (amplificación de corriente)}

La ganancia es simplemente la relación de la corriente de salida del ánodo a la corriente fotoeléctrica procedente del fotocátodo. Idealmente, la ganancia del PMT se define como $\delta^{n}$ [9], donde $n$ es el número de etapas y $\delta$ es la relación de emisión de electrones secundarios y viene dada por

$$
\delta=A E^{\alpha} \text {. }
$$

El parámetro $A$ es una constante de proporcionalidad que varía con el tipo de PMT, $E$ es el voltaje entre etapas, y $\alpha$ es un coeficiente determinado por el material de los dínodos y la estructura geométrica. Por lo general, $\alpha$ tiene un valor entre 0,7 a 0,8

Cuando se aplica un voltaje V entre el cátodo y el ánodo de un PMT compuesto por $n$ etapas de dínodos, la ganancia $G$ se convierte en

$$
\begin{gathered}
G=\delta^{n}=\left(A E^{\alpha}\right)^{n}=\left\{A\left(\frac{V}{1+n}\right)^{\alpha}\right\}^{n} \\
=\frac{A^{n}}{(1+n)^{\alpha n}} V^{\alpha n}=K V^{\alpha n}
\end{gathered}
$$

Dado que en general los PMT tienen de 8 a 12 etapas de dínodos, la salida del ánodo varía directamente con la potencia 6 a 10 del cambio en el voltaje aplicado. Además, la señal de salida del PMT es extremadamente susceptible a las fluctuaciones de la tensión de alimentación, por lo tanto la fuente de alimentación debe ser muy estable y exhibir ondulaciones (ripple) mínimas.

\section{II-B. Linealidad}

La relación entre el número de fotones incidentes al número de electrones recogidos en el ánodo se denomina linealidad de carga. A la proporcionalidad entre el flujo incidente y la corriente de ánodo se le llama linealidad de 
corriente. En esta última relación, por lo tanto, el tiempo es un parámetro adicional. Existen factores internos y externos que afectan tanto la linealidad de carga como la linealidad de corriente y su estudio escapa a los objetivos de este artículo. Para un tratamiento más extenso del tema se recomienda la lectura de la referencia [9].

A efectos de fijar criterios, en este artículo la linealidad hace referencia a la relación $i_{a} / i_{k}$ entre la corriente de ánodo y la corriente de cátodo; la cual, bajo condiciones ideales es constante e independiente del valor de $i_{a}$.

Una cuestión importante a considerar cuando se estudia la linealidad es el "efecto de carga espacial". Cuando los electrones se liberan de un dínodo, no van a salir todos a la vez, ni van a tener la misma energía cinética. Un grupo de electrones, por lo tanto, se propagará en el espacio y llegará al siguiente dínodo en diferentes momentos. Los electrones tienen carga negativa y van a crear un campo eléctrico en oposición al campo (positivo) entre los dos dínodos en cuestión. En pequeñas cantidades el campo eléctrico creado por los electrones que llegan en primer lugar crearán sólo un campo pequeño de oposición. Los electrones que vayan llegando sucesivamente tendrán suficiente energía cinética para superar fácilmente esa oposición. Pero, en números más grandes, los electrones que llegan primeros pueden crear un campo opuesto lo suficientemente grande como para inhibir la llegada de los electrones finales. Este efecto puede evitar que el PMT opere como un instrumento lineal; la ganancia del PMT con niveles de entrada de luz elevados se ve disminuida en comparación con la ganancia para niveles de luz más bajos. Los lugares más probables para que esto ocurra son las etapas finales del PMT donde el número de electrones es mayor. Una manera simple de corregirlo es aumentar las tensiones inter-dínodos en las últimas etapas. El aumento resultante en la energía cinética de los electrones es a menudo suficiente para superar el campo de oposición. Esta intensidad de campo adicional, sin embargo, también contribuye a disminuir el tiempo de vida útil del PMT, los dínodos finales se desgastan con mayor rapidez debido a las interacciones adicionales de electrones.

El rango en el que el PMT se desempeña de manera lineal depende del material de los dínodos así como del tipo de estructura del fotomultiplicador. Todos los fotomultiplicadores ofrecen una mejora en la linelidad cuando son operados con divisores de tensión que entregan tensiones más elevadas en los últimos tres o cuatro dínodos.

En aplicaciones pulsadas, se acostumbra entregar valores típicos de linealidad de pulso especificados en dos puntos (desviaciones de la proporcionalidad lineal del $\pm 2 \%$ y $\pm 5 \%)$. En esta medición se suele emplear la técnica de los dos pulsos. Se utilizan dos LEDs como fuente de luz pulsada. Dos pulsos son aplicados, uno retrasado con respecto al otro, luego los dos pulsos se aplican en coincidencia (salida sumada). La no linealidad se hace evidente cuando el pulso de salida sumado difiere de la suma esperada de los pulsos individuales. El ancho de los pulsos aplicados es del orden de $50 \mathrm{~ns}$ y la tasa de repetición es normalmente de $1 \mathrm{kHz}$.

\section{LOS CENTELLADORES}

Debido a que los materiales centelladores son de uso muy común en conjunto con los tubos fotomultiplicadores, conviene aquí centrar la atención en las características que serán útiles al momento de entender su funcionamiento y su respuesta, y cómo afectarán a la respuesta del PMT.

Los materiales centelladores, en términos generales, se dividen en dos clases: centelladores orgánicos e inorgánicos. La física del mecanismo de centelleo, las propiedades y las aplicaciones de ambos tipos de materiales centellantes son muy diferentes. Desde el punto de vista de la aplicación la diferencia importante es que los centelladores orgánicos contienen, en su mayor parte, átomos con un número atómico $\mathrm{Z}$ pequeño, y entregan longitudes de radiación mayores. Los centelladores inorgánicos son interesantes, principalmente debido a que muchos de ellos contienen una gran fracción de átomos con un número atómico $\mathrm{Z}$ alto y por lo tanto estos materiales entregan una radiación de longitud más corta. Debido a esta diferencia de longitud de la radiación, los centelladores inorgánicos se utilizan principalmente para detección de rayos gamma y rayos $\mathrm{X}$, mientras que los centelladores orgánicos se utilizan principalmente en detección de partículas cargadas rápidas. El punto importante aquí es que para obtener una buena eficiencia de detección, el máximo de emisión del centellador debe coincidir (o estar muy próximo) con el punto de máxima sensibilidad espectral del PMT elegido para la aplicación, ya que a pesar de que se utilicen centelladores de gran área/volumen, apenas una fracción de la luz es recogida por el PMT (aproximadamente entre $10 \%$ y $25 \%$ ). Se recomienda al lector interesado en obtener mayor información sobre los materiales centelladores consultar la bibliografía especializada [1].

En general se puede considerar que el perfil temporal del pulso de luz entregado por un centellador consiste en una señal con una subida muy rápida seguida de un decaimiento exponencial como el descrito por (3),

$$
I=I_{0} e^{-\left(t / \tau_{s}\right)},
$$

donde $\tau_{s}$ representa el tiempo de decaimiento que caracteriza la respuesta de los diferentes materiales centellantes e $I_{0}$ es el pico de intensidad alcanzado luego de la subida rápida. Para los centelladores rápidos, el tiempo de decaimiento ronda los 2 ns de duración. En la actualidad se acostumbra especificar el desempeño de los centelladores orgánicos ultra-rápidos a través de su tiempo $\mathrm{FWHM}^{2}$ en lugar de sólo indicar su tiempo de decaimiento. Como ejemplo, en la siguiente tabla se pueden ver las características temporales de un centellador orgánico.

\begin{tabular}{cc} 
Propiedad del Centellador & Valor \\
\hline Tiempo de crecimiento (ns) & 0.9 \\
Tiempo de decaimiento (ns) & 2.1 \\
Ancho del pulso (FWHM, ns) & $\sim 2.5$ \\
IV. EL CIRCUITO EQUIVALENTE DEL TUBO \\
FOTOMULTIPLICADOR Y SU SEÑAL DE SALIDA
\end{tabular}

Para diseñar y analizar los circuito electrónicos de polarización y amplificación a utilizar con el PMT es necesario

${ }^{2}$ La anchura a media altura, abreviada FWHM (del inglés Full Width at Half Maximum) es una medida de la extensión de una función, que viene dada por la diferencia entre los dos valores extremos de la variable independiente en los que la variable dependiente es igual a la mitad de su valor máximo. 
tener un circuito equivalente que posea los elementos de circuito mínimos con los cuales imitar su comportamiento. De manera similar, para describir la señal de salida, resulta práctico usar una representación funcional que sea simple pero adecuada.

En un PMT la ganancia se genera por emisión secundaria, tal como se comentó en la Sección I, y por lo tanto el circuito equivalente es el de un generador de corriente ideal $i(t)$, en paralelo con una resistencia $R_{0}$ y una capacitancia $C_{0}$ de salida, tal como muestra la Figura 3. Este circuito es suficiente para la mayoría de las aplicaciones, e igualmente válido para corriente directa (cd) o aplicaciones de señales pulsadas. $C_{0}$ es la capacitancia entre el ánodo y tierra del circuito. Su magnitud depende principalmente del área de los dínodos y de la separación entre los mismos: para tubos de diámetros inferiores a $25 \mathrm{~mm}, C_{0} \sim 3 \mathrm{pF}$ y para aquellos de diámetros superiores a $50 \mathrm{~mm}, C_{0}$ puede exceder los $10 \mathrm{pF}$. Los conductores dentro del PMT agregan alguna inductancia (del orden de $100 \mathrm{nH}$ ) cuyo efecto a veces se observa como una oscilación de alta frecuencia en la parte final de los pulsos de salida. Este efecto puede evitarse agregando resistencias de amortiguamiento en serie con cada uno de los dos o tres últimos dínodos en la red de división de tensión. De esta forma se evita la complicación del circuito equivalente por la inclusión de inductancias.

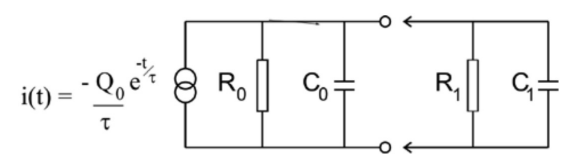

Figura 3. El circuito equivalente de un PMT está compuesto por un generador de corriente en paralelo con $R_{0}>>10^{9} \Omega$ y $C_{0} \sim 2-20 \mathrm{pF}$. La mayoría de las aplicaciones se pueden analizar en términos de una combinación de $\mathrm{R}$ y C. Sin pérdida de generalidadd, podemos tomar $R=$ $R_{0} / / R_{1}$ y $C=C_{0}+C_{1}$. Con $R_{1}$ y $C_{1}$ las resistencia y capacitancia equivalentes del circuito de carga.

La forma de un pulso de salida iniciado por un único fotoelectrón, tal como se puede observar en el ánodo de un PMT, contiene toda la información necesaria para el análisis del circuito. Debido a que cualquier señal multi-fotoelectrón se puede considerar como la superposición de un conjunto de eventos generados por fotoelectrones simples, con una distribución acotada en el tiempo, todo lo que se necesita saber es $i(t)$ para un solo fotoelectrón de excitación. La señal de salida de los PMT tienen tiempos de subida que van desde $1 \mathrm{~ns}$ a aproximadamente $10 \mathrm{~ns}$, con tiempos de decaimiento unas 2 a 3 veces el tiempo de subida.

El siguiente análisis muestra cómo la capacitancia de salida junto con la resistencia de carga del ánodo determina la naturaleza de la señal de salida para un PMT. Sin alejarse demasiado de la realidad, se puede considerar como entrada un pulso de luz proveniente de un centellador con decaimiento exponencial en la intensidad, con una constante de tiempo $\tau_{s}$, tal como la representada en (3). Por consiguiente, la corriente de fotoelectrones $i(t)$ viene dada por:

$$
i(t)=-\frac{N q}{\tau_{s}} e^{-\left(t / \tau_{s}\right)}
$$

donde $\mathrm{N}$ es el número total de fotoelectrones y $q=1.6 \times$ $10^{-19} \mathrm{C}$ es la carga de un electrón. Para un PMT ideal de ganancia $\mathrm{G}$, la corriente de salida viene dada por

$$
I(t)=G i(t),
$$

de acuerdo a esto, se tiene que la tensión de salida para el circuito de la Figura 3 puede expresarse como

$$
V_{o}(t)=-\frac{G N q R}{\left(\tau_{s}-\tau\right)}\left[e^{-\left(t / \tau_{s}\right)}-e^{-(t / \tau)}\right] \quad \forall \tau_{s} \neq \tau
$$

donde $\tau=R C$ es la constante de tiempo de salida, la cual incluye la resistencia de carga de ánodo y cualquier combinación de $\mathrm{R}$ y $\mathrm{C}$ en paralelo acoplado a la salida del PMT. La elección $\tau=\tau_{s}=R C$ no es inusual y para ese caso se debe tomar el límite $\tau \rightarrow \tau_{s}$ en (6) para obtener

$$
V_{o}(t)=-\frac{G N q R t}{\left(\tau_{s}^{2}\right)} e^{-\left(t / \tau_{s}\right)},
$$

o en función de C,

$$
V_{o}(t)=-\frac{G N q t}{\left(C \tau_{s}\right)} e^{-\left(t / \tau_{s}\right)}
$$

Las ecuaciones (6) a (8) caracterizan el circuito más simple que se puede utilizar con un tubo fotomultiplicador, una resistencia. Aquí la resistencia realiza la función de conversión de corriente a voltaje. La ecuación (6) se refiere al pulso de salida en el caso ideal, mientras que en la práctica la dispersión del tiempo de tránsito ${ }^{3}$ de las partículas cargadas en el PMT también afecta la forma del pulso. Sin embargo, aceptando esta limitación, dicha ecuación puede usarse para predecir la señal de salida en la mayoría de los casos.

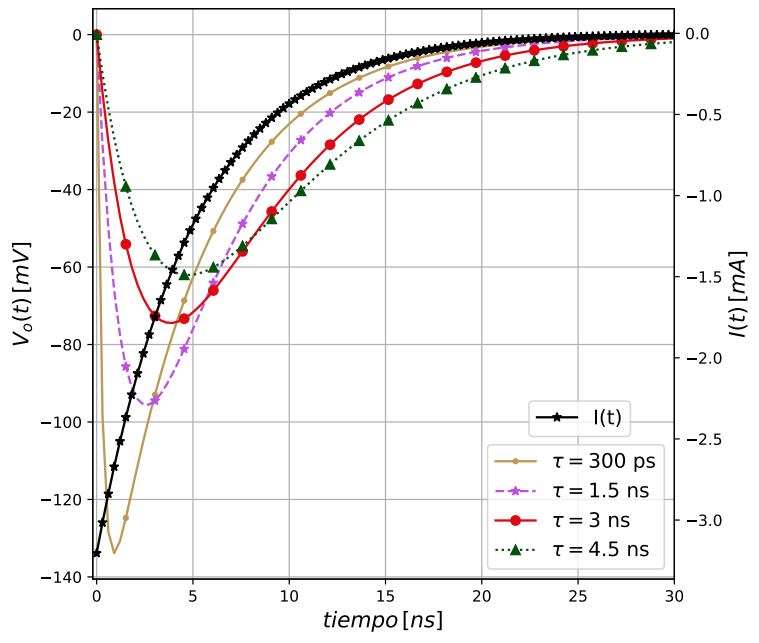

Figura 4. Pulsos de salida con $\tau_{s}=5 \mathrm{~ns}$, resistencia de carga de ánodo $R=50 \Omega, G=1 \times 10^{6}, N=100$ y un rango de capacidades. El agregado de capacitancia en paralelo causa la disminución de la altura de los pulsos de salida.

Al analizar las ecuaciones (7) y (8), y su representación gráfica en las Figuras 4 y 5 , se pueden obtener varias conclusiones respecto de la señal de salida del PMT.

- La máxima amplitud de señal se obtiene para $R \rightarrow$ $\infty$. En este caso la corriente $I(t)$ simplemente estará cargando el capacitor $\mathrm{C}$ y la tensión de salida será

$$
V_{o}(t)=-\frac{G N q}{C}\left[e^{-\left(t / \tau_{s}\right)}-1\right]
$$

${ }^{3} \mathrm{El}$ intervalo entre la llegada de un pulso de luz al cátodo y el del pulso de corriente correspondiente al ánodo se llama tiempo de tránsito. 


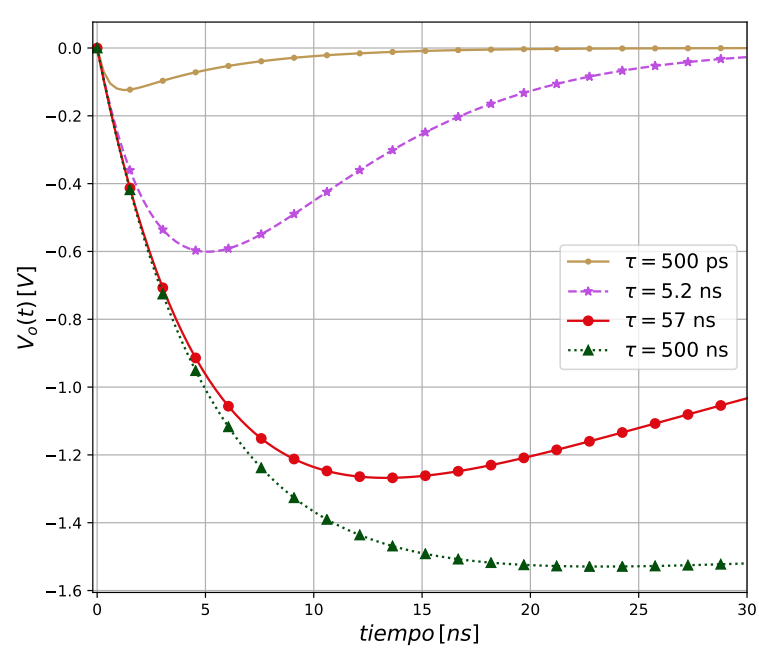

Figura 5. Tensión de salida para una capacitancia de carga $C=10 \mathrm{pF}$, $\tau_{s}=5 \mathrm{~ns}, G=1 \times 10^{6}, N=100$ y un rango de resistencias de carga. La señal de salida ya no sigue la excitación cuando $\tau>>\tau_{s}$.

- Solamente cuando $\tau<<\tau_{s}$ el voltaje de salida será una reproducción de la corriente $I(t)$ de entrada.

- El área bajo los pulsos es la misma y proporcional a GNq.

- Al considerar el caso $\tau=\tau_{s}$, cuando $I(t)$ ha decaído hasta el $1 \%$ de su valor inicial, $V_{o}(t)$ es todavía $10 \%$ de $V_{\max }$. En otras palabras, el pulso de salida tendrá un decaimiento largo, que aumenta con $\tau$.

- Cuando $R \leq 100 \Omega$, lo que implica $\tau<<\tau_{s}$, el pulso no se integra - esto se denomina "funcionamiento en modo corriente" -, con el tiempo de subida de los pulsos de corriente y de tensión determinado principalmente por la mayor constante de tiempo. Con $\tau>>\tau_{s}$, el pulso de corriente se integra y esta forma de funcionamiento se denomina "modo de tensión". Se puede notar cómo el tiempo de subida (rise time) aumenta a medida que se alcanza el "modo de tensión" y, en el caso extremo, con $\tau \rightarrow \infty$, el tiempo de subida de la tensión es igual al tiempo de decaimiento de la entrada.

- Si la tasa de eventos es $\sim 1 / \tau$, ocurre el fenómeno de acumulamiento ("pulse pile-up") [10].

Del análisis anterior, si la forma de los pulsos es lo importante, entonces la capacitancia de salida del PMT además de las capacidades parásitas pondrán un límite al valor máximo de resistencia de carga a utilizar y por lo tanto a la ganancia efectiva de tensión. Este tipo de problemas se pueden solucionar, en parte, mediante el uso de amplificadores rápidos realimentados.

\section{IV-A. La influencia de los capacitores de acople}

Antes de continuar con el diseño de la red de polarización, resulta de interés conocer un poco más sobre la influencia que tienen los capacitores de acople en la señal de salida.

Si bien anteriormente se había indicado que el voltaje de salida es una reproducción fiel del pulso de entrada cuando $C R<<\tau_{s}$, o lo que es equivalente, $C R<<t_{p}$ ( $t_{p}$ es en este caso el ancho FWHM del pulso de corriente), nunca es posible la transmisión libre de distorsiones: al elegir $C R<<t_{p}$ se logra la recuperación rápida de la línea de base, pero con una amortiguación pobre en la señal de salida; con $C R>t_{p}$ se logra una larga recuperación hasta el cero con un mayor amortiguamiento en la señal. El capacitor de acoplamiento hace que la línea de base, o nivel de referencia de la señal de salida cambie con la velocidad y la amplitud del pulso. Interpretar el efecto combinado del capacitor de acople y el resto de las capacitancias del circuito es claramente complicado en el caso general, ya que el circuito equivalente se caracteriza por tres constantes de tiempo y la respuesta es sensible a la tasa de eventos. Como regla general, suele tomarse $C_{\text {acople }}>C_{i n}$; con $C_{i n}$ la capacitancia total de entrada del circuito equivalente visto desde la carga. Un valor razonable puede ser $C_{\text {acople }}=100 C_{i n}$, para poder considerar que $C_{a c o p l e}$ es un cortocircuito para la transmisión de señales.

La elección de $C_{\text {acople }}$ va a ser siempre un compromiso entre respuesta rápida y baja distorsión de la señal. El valor final en general saldrá de pruebas realizadas en el circuito.

\section{DISEÑO DEL DIVISOR DE TENSIÓN}

\section{$V$-A. Cálculo de la corriente media de ánodo}

En el diseño de la red divisora de tensión para el PMT, un parámetro que importa es la corriente media de ánodo esperada en funcionamiento normal. Este parámetro se toma como referencia para hacer los cálculos de las corrientes que circularán por el divisor y por el PMT y sirve para determinar el rango de trabajo donde se asegura cierto grado de linealidad del circuito.

La corriente media de ánodo del PMT, $I_{m e d P M T}$, es el producto de la carga por pulso, $Q$, tal como se vio en la Sección IV, y la frecuencia de pulsos (que es la misma que la tasa de conteo), $f_{p}$,

$$
I_{m e d P M T}=Q f_{p}
$$

Con el fin de asegurar que las variaciones de tensión debido a variaciones de la corriente de ánodo sean despreciables, la corriente nominal del divisor $I_{d}$ debe ser mucho mayor que la corriente de ánodo, $I_{a}$. Para asegurar esta condición, en la práctica se acostumbra hacer los cálculos aplicando la siguiente regla:

$$
I_{d} / I_{a} \geq 100
$$

La corriente a utilizar en los cálculos para $I_{a}$ en (11) es la corriente media de ánodo prevista para la aplicación; tanto para operación con corriente directa como para aplicaciones pulsadas.

\section{V-B. La teoría del diseño}

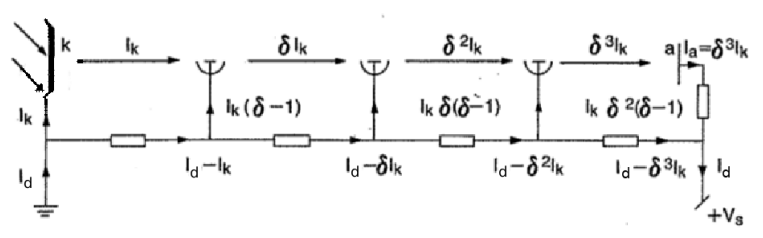

Figura 6. Esquema idealizado de un PMT de tres etapas y las corrientes involucradas en cada etapa. 
Se puede observar un esquema idealizado de PMT en la Figura 6 y analizar lo que sucede cuando este circuito está en funcionamiento. Si se tienen $\mathrm{N}$ electrones por segundo en la entrada, éstos generan una corriente de cátodo $I_{k}$, que luego de ser amplificada por 3 etapas, cada una de ganancia $\delta$, aparece como una corriente de salida. Un incremento en $I_{k}$ causa una caída en la tensión $\Delta V$ entre el tercer dínodo, $d_{3}$, y el ánodo y, debido a que la tensión total aplicada es constante, $\Delta V$ aparecerá como un incremento positivo en las etapas iniciales, $d_{1} \mathrm{y} d_{2}$. El efecto se traduce como una variación en la ganancia total del dispositivo debido a que la característica de ganancia de los dínodos sigue una ley de potencias con la tensión aplicada (véase la Sección II-A). La clave para que el diseño del divisor de tensión sea exitoso radica en minimizar ese efecto de realimentación.

Resulta conveniente considerar dos casos distintos:

\section{a) Operación con corriente directa}

Si $I_{k}$ es continua, o de variación lenta, entonces se requiere para un tubo de $n$ etapas:

$$
I_{d}-I_{k} \delta^{n}=I_{d}-I_{a} \simeq I_{d},
$$

donde $I_{d}$ es la corriente que circula a través de la fuente de alta tensión. La ecuación (12) se satisface si $I_{d} \gg I_{a(\max )}$ tal que las corrientes individuales de cada resistor $I_{d}-I_{k} \delta^{n}, I_{d}-I_{k} \delta$ y $I_{d}-I_{k}$ son aproximadamente $I_{d}$. Satisfacer (12) asegura que las tensiones inter-dínodo y por lo tanto la ganancia permanecerá esencialmente constante para $I_{a} \leq I_{a(\max )}$.

b) Aplicaciones pulsadas o transitorias

Si $\hat{i}_{a}$ es una corriente transitoria, entonces resulta necesario mantener constantes las tensiones inter-dínodos por un tiempo igual a la duración de $\hat{i}_{a}$. Esto puede hacerse usando capacitores de desacople inter-dínodos para entregar el exceso de carga requerida por los pulsos. En aplicaciones pulsadas, por lo tanto, se puede tener:

$$
I_{d} \ll \hat{i}_{a} \text {, siempre que } I_{d} \gg \bar{I}_{a}
$$

donde $\bar{I}_{a}$ es la corriente media derivada de $\hat{i}_{a}$ integrada en un determinado período de tiempo (véase la ecuación (10)).

V-C. Consideraciones para la región cátodo - primer dínodo $\left(V_{k-d_{1}}\right)$

La interfase entre el fotocátodo y la entrada a las etapas multiplicadoras de electrones es una región crítica del fotomultiplicador. La distribución de la tensión entre el cátodo, electrodo de enfoque y primer dínodo tiene influencia sobre la eficiencia de recolección de fotoelectrones y la relación de emisión secundaria del primer dínodo. Estos parámetros son los principales factores en la determinación de la relación señal a ruido de salida, la dispersión de la altura de pulsos en las regiones de fotones individuales y fotones múltiples, y también la dispersión de tiempo de tránsito de los electrones. Además, la distribución de la tensión en las primeras etapas afecta a la linealidad del cátodo, la resolución de energía en el conteo de fotones y las características magnéticas del tubo fotomultiplicador, y por lo tanto su ajuste requiere especial atención, al igual que en el caso de las últimas etapas.

Algunos tubos incluyen elementos de enfoque para mejorar la eficiencia en la recolección de electrones y para minimizar las variaciones de tiempo de tránsito de aquellas señales originadas en diferentes regiones de la superficie del fotocátodo. Sin embargo, la mayoría de los PMTs no tienen elementos de enfoque ajustable - están internamente conectados al cátodo o a $d_{1}$ o totalmente ausentes -. Para este último grupo suele ser conveniente seguir las recomendaciones que entregan los fabricantes respecto de la tensión a aplicar en esa primer región. El desempeño de los tubos con elementos de enfoque separado se optimiza configurando el voltaje de enfoque experimentalmente. En algunos casos se suelen utilizar diodos Zener para mantener constante esa tensión de enfoque. En cualquier caso, de acuerdo a lo que requiera la aplicación, uniformidad de recolección o velocidad de respuesta, la tensión aplicada en esa primer región es determinada experimentalmente.

\section{V-D. Aplicaciones pulsadas}

Se llama divisor uniforme a aquel en el que todas las resistencias que lo componen son iguales. Los divisores uniformes son adecuados para funcionar en aplicaciones pulsadas siempre que la corriente de pico $\hat{i}_{a}$ de ánodo satisfaga:

$$
\hat{i} \ll I_{d}
$$

Es posible manejar pulsos de corriente mucho más grandes que $I_{d}$ modificando el circuito uniforme incluyendo capacitores de desacople en las etapas finales. En este tipo de divisores se puede tener

$$
\hat{i} \gg I_{d}
$$

siempre que se mantenga la condición (11).

Resulta aceptable operar con $\hat{i}_{a} \simeq 100 I_{d}$ siempre que la tasa de conteo no sea excesivamente alta. Si este es el caso, la condición (11) ya no será válida y la ganancia variará con la tasa de conteo.

La capacitancia requerida puede estimarse a partir de la ecuación que relaciona la tensión, la carga y la capacitancia, $Q=C V$. Si se considera un cambio pequeño en la tensión inter-dínodos, $\Delta V$, debido a un cambio en la carga $\Delta Q$, se puede obtener la caída relativa de tensión $\Delta V / V$ como

$$
\frac{\Delta V}{V}=\frac{1}{C} \frac{\Delta Q}{V}
$$

Tomando a $Q$ como la carga del pulso de salida, entonces para que la ganancia permanezca estable, $\Delta V / V$ debería permanecer $<0.01 \%$ de modo que

$$
C=\frac{\frac{\Delta Q}{V}}{\frac{\Delta V}{V}}
$$

El valor del capacitor que desacopla $d_{n}$ y $d_{n-1}$ podría reducirse debido a que la carga que se le suministra es menor por un factor $\delta$, la ganancia de la etapa. Lo mismo aplica para la etapa $d_{n-1}$ y $d_{n-2}$, pero en la práctica se acostumbra usar el mismo valor de capacitancia por conveniencia.

\section{V-E. Circuitos divisores de tensión cónicos}

En aplicaciones de medición de luz pulsada normalmente es necesario mejorar el gradiente de voltaje en las últimas etapas del circuito divisor de tensión mediante el uso de resistencias de mayor valor. Este tipo de divisores de tensión se llaman cónicos y resultan eficaces en la mejora de varias 


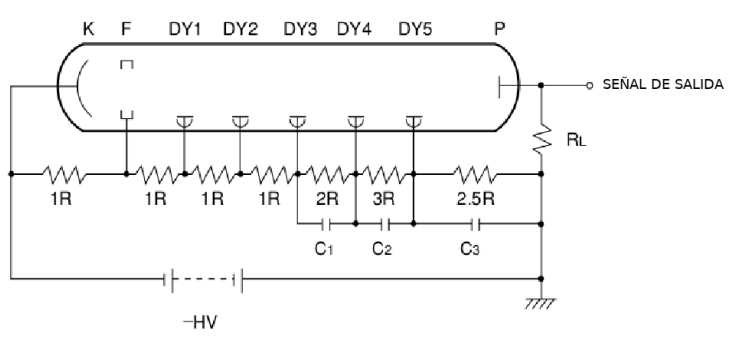

Figura 7. Circuito en configuración cónica como medida para mejorar la linealidad en modo pulsado. El terminal $\mathrm{F}$ corresponde al electrodo de enfoque. $K$ corresponde al fotocátodo, $D Y 1, D Y 2, \ldots D Y 5$ son los terminales de dínodos y $P$ es el ánodo.

características. Se debe tener en cuenta, sin embargo, que cuando se utiliza este tipo de circuito divisor, la ganancia del conjunto disminuye a medida que el gradiente de voltaje es mayor; aproximadamente $1 / 3$ a $1 / 5$ en comparación con la salida del ánodo del divisor de tensión uniforme. Por lo tanto, se requiere un ajuste para aumentar la tensión de alimentación del tubo fotomultiplicador. Adicionalmente, se requiere especial atención con respecto a la tolerancia de tensión entre etapas del PMT a medida que se suministra un voltaje más alto.

La Figura 7 muestra un circuito divisor de tensión cónico utilizado para un PMT de 5 etapas. En este circuito, la tensión DY5 - ánodo se fija en un valor inferior a la tensión DY4-DY5. Esto es así porque la distancia de los electrodos entre el último dínodo y el ánodo es generalmente corta, de manera que se puede conseguir un gradiente de tensión adecuado aplicando una tensión relativamente más baja.

\section{V-F. Red divisora de tensión activa}

Para mejorar la estabilidad de las tensiones en las últimas etapas, se puede utilizar transistores en lugar de sólo resistencias, tal como se muestra en la Figura 8. El uso de una red resistiva estándar limita la corriente de ánodo a un pequeño porcentaje de la corriente del divisor, de manera que las caídas de tensión en las últimas etapas son insignificantes. Sin embargo, el uso de transistores permite que la corriente media de ánodo pueda llegar a valores tan altos como la mitad de la corriente que circula por el divisor.

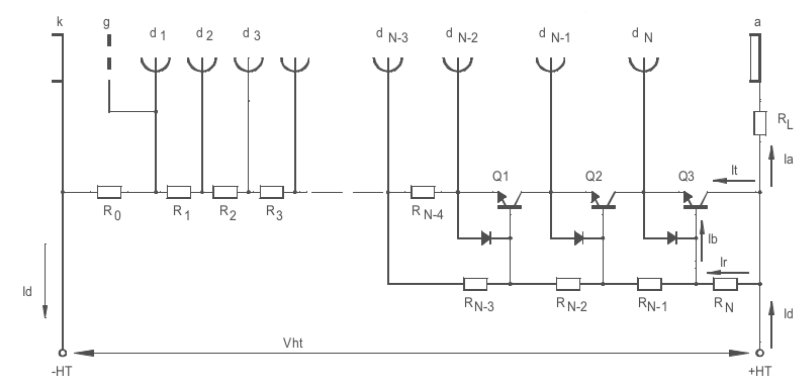

Figura 8. Uso de transistores en la red divisora para mantener constantes las tensiones inter-dínodos. Este esquema se puede utilizar donde son necesarias altas tasas de conteo. Los diodos protegen a los transistores de eventuales tensiones inversas aplicadas sobre los mismos.

Observando la Figura 8 podemos hacer el siguiente análisis. La corriente del divisor, $I_{d}$, fluye a través de los resistores en serie $R_{0}$ a $R_{3}$ de la red resistiva antes de dividirse en dos partes, de acuerdo con los valores de las resistencias $R_{N-4}$ y $R_{N-3}$ (debido a que la tensión base-emisor del transistor $Q_{1}$ es aproximadamente cero voltios, $R_{N-4}$ y $R_{N-3}$ están prácticamente en paralelo). Para simplificar, se puede suponer que ambas resistencias tienen el mismo valor, entonces $I_{d} / 2$ fluye a través de $R_{N-4}$ y la cadena de transistores, e $I_{d} / 2$ también fluye a través de $R_{N-3}$ hasta $R_{N}\left(I_{t} \cong I_{r}\right)$. Los transistores se utilizan como seguidores de emisor, lo que significa que sus voltajes de emisor, y por lo tanto las tensiones entre las últimas etapas, siguen las tensiones de base. En otras palabras, las tensiones entre etapas serán estables siempre que las tensiones de base sean estables, lo cual es cierto si los transistores tienen alta ganancia $\left(h_{f e}>50\right)$. Así, las corrientes de base, $I_{b}$, son insignificantes en comparación con $I_{r}$.

Los transistores actúan como fuentes de tensión, independientemente de la corriente de colector, $I_{t}$. El valor de $I_{t}$ oscila entre $I_{d} / 2$, cuando la corriente de ánodo es cero, hasta prácticamente cero cuando la corriente del ánodo se aproxima a $I_{d} / 2$. El valor de estado estacionario de $I_{t}$ determina la $I_{a}$ máxima absoluta que el diseño puede manejar. Es recomendable dejar un margen de seguridad, para evitar que $I_{a}$ alcance el valor de $I_{t}$.

En función de la corriente de ánodo promedio requerida, se pueden colocar transistores en 2, 3 o más etapas, debido a que las tensiones entre las últimas etapas son las más afectadas por la corriente del ánodo. Cada diseño se puede optimizar también mediante la experimentación con diferentes proporciones de resistencias $R_{N-4} / R_{N-3}$. El circuito también funciona como limitador de corriente, que previene de daños al tubo fotomultiplicador.

\section{V-G. La resistencia de ánodo}

Ya sea que el PMT esté polarizado con una fuente positiva o negativa respecto a tierra, el potencial del ánodo debe permanecer fijo. Siempre debe conectarse una resistencia de protección entre el ánodo y la tierra del circuito. La resistencia de protección debe ser alta debido a que se encuentra conectada en paralelo con la alta resistencia interna del PMT. El valor elegido depende principalmente del circuito de carga y es típicamente $\geq 10 \mathrm{k} \Omega$.

Si el tubo está conectado en polaridad positiva y acoplado capacitivamente a la circuitería de medición, es esencial una resistencia entre el ánodo y el terminal positivo de la fuente de alto voltaje; esta resistencia también puede constituir la carga de ánodo. Una vez más, la resistencia debe ser razonablemente alta y depende principalmente de la impedancia de entrada del circuito de medición de la siguiente etapa.

\section{V-H. Señal del último dínodo}

Normalmente se toma la señal de salida del ánodo para analizar los eventos producidos en el detector; pero en ciertas aplicaciones resulta útil disponer de la señal de salida del último dínodo, antes del ánodo.

Se elige la señal del último dínodo debido a que:

- La señal del dínodo se produce simultáneamente con la salida del ánodo, de modo que se puede utilizar como señal de temporización de eventos. 
- Puede utilizarse en conjunto con la señal del ánodo para extender el rango dinámico de la medición.

- La señal tomada del último dínodo tiene una amplitud comparable con la del ánodo y tiene una mayor relación señal/ruido que los otros dínodos.

- La señal del último dínodo tiene polaridad opuesta a la del ánodo.

El divisor de tensión uniforme debe modificarse con el fin de leer la señal del último dínodo. El principal problema con esta modificación es cómo obtener esa señal sin perturbar la del ánodo. Para mantener constantes las tensiones interdínodos se suele agregar capacitores de desacople en serie.

Cuando se utiliza cable coaxial de $50 \Omega$ de impedancia característica para transmitir la señal del dínodo, se debe conectar una resistencia de $200 \Omega$ a $300 \Omega$ antes de la salida (entre el último dínodo y su salida) con el fin de amortiguar las oscilaciones que aparecen en la señal de salida por efecto de la desadaptación de impedancias. Lo mismo aplica para cuando se conecta una etapa amplificadora de baja resistencia efectiva de entrada, como puede ser un amplificador de transimpedancia.

Debido a que el último dínodo todavía tiene un potencial más negativo que el ánodo, su salida se suele acoplar con un capacitor de alta tensión, permitiendo únicamente el paso de las señales transitorias.

\section{APLICACIÓN}

En la Figura 9 se muestra un ejemplo de mejora que puede lograrse en la linealidad y en la extensión del rango dinámico de la señal de salida de un PMT gracias al uso de un divisor activo, siguiendo los lineamientos de diseño esbozados en este artículo.

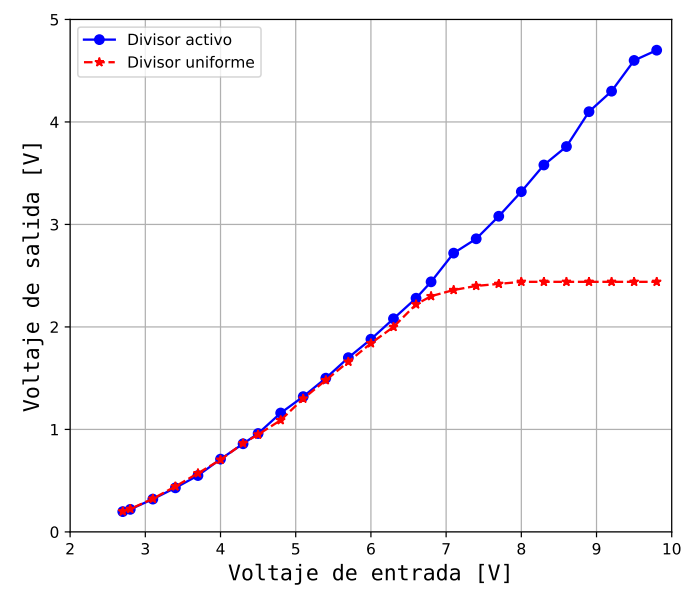

Figura 9. Mejora en la extensión del rango dinámico y linealidad de la señal de salida de un PMT modelo R1463. La mejora corresponde al uso de un divisor activo, diseñado para conteo de fotones. Se observa un efecto de saturación en el divisor uniforme con corrientes grandes $\left(I_{a}>40 \mathrm{~mA}\right)$ en la señal de salida.

La medición corresponde a la aplicación de pulsos de luz a un PMT modelo R1463, de Hamamatsu. El PMT se utiliza junto a un centellador en el conteo de fotones para la detección de partículas. Este tipo de aplicaciones requiere buena linealidad para altas tasas de eventos $\left(f_{p} \sim 1 \mathrm{kHz}\right)$ y para un gran rango de intensidades, ya que a partir de la amplitud y forma de los pulsos de salida se determina la energía de las partículas incidentes.

Con el fin de estudiar la respuesta del PMT a los centelladores, se armó un arreglo experimental para entregar al PMT pulsos de luz de forma controlada y similares a los que se obtienen en un centellador. Se utilizó un divisor uniforme y un divisor activo con una carga de $50 \Omega$ en ambos casos. Los pulsos de luz provienen de un LED modelo L$7113 \mathrm{NBC}$, el cual entrega fotones de $\lambda=445 \mathrm{~nm}$ de forma predominante. El ancho de los pulsos es del orden de los $8 \mathrm{~ns}$ y la tasa de repetición utilizada fue de $1 \mathrm{kHz}$. El voltaje de entrada corresponde a la amplitud máxima de los pulsos aplicados al circuito de manejo del LED. La tensión de salida corresponde a la tensión pico medida en la carga. Para obtener la tensión de salida, se hace un promedio de 1000 pulsos aplicados para cada una de las tensiones de entrada.

\section{CONCLUSIONES}

En este artículo se analizaron los factores que afectan a la formación de la señal, y cuáles son los recaudos a tener en cuenta cuando se procesan los pulsos de salida de un tubo fotomultiplicador. Se estudió el circuito equivalente y la red de polarización de estos dispositivos. Las aplicaciones incluyen desde el conteo de fotones para detección de partículas hasta los estudios de dispersión Raman para el análisis molecular. Se analizó, además, la interacción que existe entre el PMT y un material centellador, comúnmente utilizados en conjunto. Se pudo demostrar la mejora en la linealidad de la señal de salida con el uso de un divisor de tensión activo.

La información suministrada en este artículo puede utilizarse como guía, no sólo para el diseño de la red de polarización, sino también como base para el diseño de la etapa posterior de procesamiento de los pulsos de salida.

\section{REFERENCIAS}

[1] G. F. Knoll, Radiation Detection and Measurement. John Wiley \& Sons, ago 2010

[2] S. Casado-Terrones, J. F. Fernández-Sánchez, A. Segura-Carretero, and A. Fernández-Gutiérrez, "Simple luminescence detectors using a light-emitting diode or a Xe lamp, optical fiber and charge-coupled device, or photomultiplier for determining proteins in capillary electrophoresis: A critical comparison," Anal. Biochem., vol. 365, no. 1, pp. 82-90, 2007.

[3] A. C. Cordeiro, F. Marchi, H. R. Gamba, J. L. Fabris, G. H. Couto, H. J. Kalinowski, and E. G. Bertogna, "Application of an Ultra-Weak Bioluminescence Measurement System for Escherichia coli Detection in Sanitary Control," J. Appl. Instrum. Control, pp. 28-33, 2001.

[4] F. Shakibaie, L. Lamard, H. Rubinsztein-Dunlop, and L. J. Walsh, "Application of Fluorescence Spectroscopy for Microbial Detection to Enhance Clinical Investigations," in Phot. Count. - Fundam. Appl. InTech, mar 2018, vol. 1, ch. 10, p. 13.

[5] P. G. Papageorgas, "A multichannel photon counting system for gas analysis with raman-scattering technique," IEEE Trans. Instrum. Meas., 1999.

[6] N. T. Quyen, M. D. Jouan, N. Q. Dao, E. Da Silva, and D. A. Phuong, "New Raman spectrometer using a digital micromirror device and a photomultiplier tube detector for rapid on-line industrial analysis. Part II: Choice of analytical methods," Appl. Spectrosc., 2008.

[7] Pierre Auger Observatory. [Online]. Available: http://auger.org.ar

[8] Hamamatsu, "R1463 Photomultiplier tube." [Online]. Available: https://www.hamamatsu.com/us/en/product/type/R1463/index.html

[9] Photonis, Photomultiplier Tubes Principles and Applications. Photonis, Brive, France, sep 2002.

[10] ORTEC, "Preamplifier introduction," Technical Note, pp. 16, 1991. [Online]. Available: http://www.ortec-online.com//media/ametekortec/other/preamplifier-introduction.pdf?la=en 